\title{
Analytical Method for Thickness and Wrinkling Measurements of 2-D Zeolites
}

\author{
Prashant Kumar, ${ }^{1}$ Kumar Varoon Agrawal, ${ }^{1}$ Michael Tsapatsis, ${ }^{1}$ K. Andre Mkhoyan ${ }^{1}$ \\ ${ }^{1}$ Department of Chemical Engineering \& Materials Science, University of Minnesota, Minneapolis, MN 55455.
}

Two-dimensional (2-D) zeolites are near single-unit-cell thick layers of silicon and oxygen atoms with precisely-sized pores of molecular dimensions [1]. It is critical to determine the thickness and structure of 2-D zeolite layers in order to understand their unique transport [2] and catalytic properties [3]. Thickness measurements of 2-D zeolites are often performed by X-ray reflectivity experiments [4] or by imaging cross-sectional samples in a transmission electron microscope (TEM) [5]. However, X-ray reflectivity measurements require fabrication of a periodic multilayer, which is not feasible for zeolite nanosheets with sub-micron lateral dimensions. On the other hand, conventional TEM (CTEM) images of cross-sections require in-depth analysis of thickness and image contrast. Due to these limitations and the electron-beam-sensitive nature of 2-D zeolites [6], a method for unambiguous determination of their thickness and structure has remained elusive.

We developed an analytical method based on a set of TEM experiments, which can provide complete quantitative characterization of 2-D zeolites, including crystal structure, uniformity, thickness, and wrinkling. Since intensity modulation of a diffraction spot on tilting is a fingerprint of the thickness, and changes in the spot shape are a measure of the wrinkling, comprehensive three-dimensional (3-D) mapping of the reciprocal lattice was performed to monitor these changes. This was achieved by acquiring a tilt series of selected area electron diffraction patterns (SAED) of MFI-structure type zeolite nanosheets on a FEI Tecnai G2 F30 (S)TEM equipped with a TWIN pole piece at $300 \mathrm{kV}$ accelerating voltage.

A MFI-nanosheet suspension in octanol was prepared by following a method reported by Varoon et al [7]. The MFI crystal structure of as-synthesized nanosheets was confirmed by high resolution conventional TEM imaging (Figure 1a) and [010] zone axis SAED pattern (Figure 1b). Comparison of multislice simulated modulation of diffraction spot intensity with tilt to experimental data revealed that the nanosheets are 1.5 unit cells (uc) thick (Figure 1c).

In order to investigate quantitatively the deviation from flatness (wrinkling) of the MFI nanosheet, we tracked changes in shape of diffraction spots upon tilting. The wrinkling of nanosheets in real space corresponds to precession of the rel-rods [8] into "cones" in reciprocal space. The diffraction spot shape changes are particularly pronounced at larger tilt angles. Quantification of these wrinkles was done by modeling two independent sine waves superposed in a- and c-directions of a flat nanosheet (Figure 1dg). Comparison with multislice simulated tilt series of SAED patterns for wrinkled nanosheet models revealed that the wrinkling is larger in the a-direction as compared to the c-direction (due to greater resistance of the MFI nanosheet to bending in c-direction). The average surface roughness of nanosheets was estimated to be $0.8 \mathrm{~nm}$.

References:

[1] M. Choi et al, Nature 461 (2009), p. 246.

[2] K. Varoon et al, AIChE. J. 59 (2013), p. 3458. 
[3] K. Na et al, J. Am. Chem. Soc. 132 (2010), p. 4169.

[4] H. Jiang et al, J. Appl. Phys. 107 (2010), p. 103523.

[5] W. Park et al, Chem. Mater. 23 (2011), p. 5131.

[6] O. Ugurlu et al, Phys. Rev. B 83 (2011), p. 113408.

[7] K. Varoon et al, Science 334 (2011), p. 72.

[8] J. C. Meyer et al, Nature 446 (2007), p. 60.

[9] This work was supported as part of the Catalysis Center for Energy Innovation, an Energy Frontier Research Center funded by the U.S. Department of Energy, Office of Science, Basic Energy Sciences under Award DE-SC0001004.

a

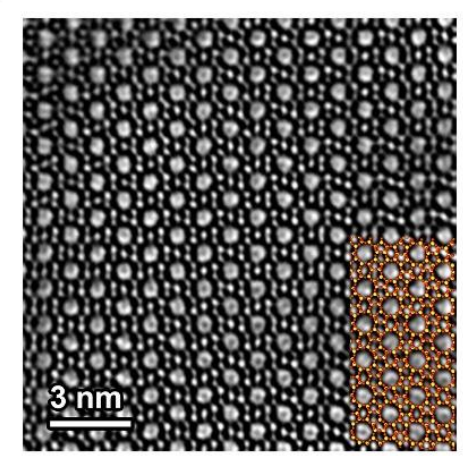

b

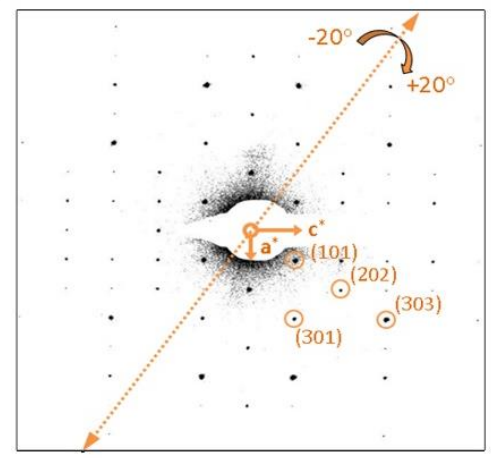

d

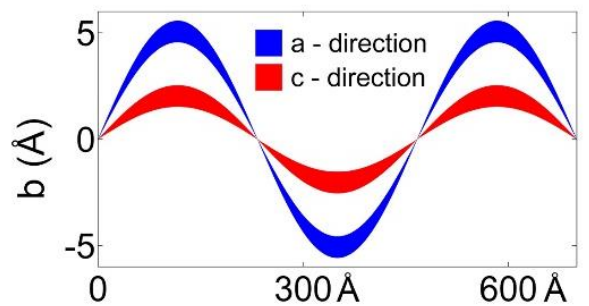

e

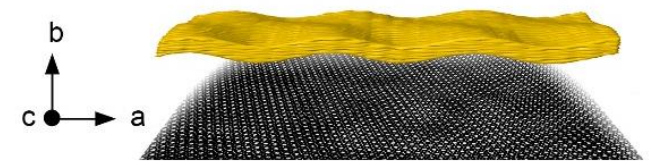

f

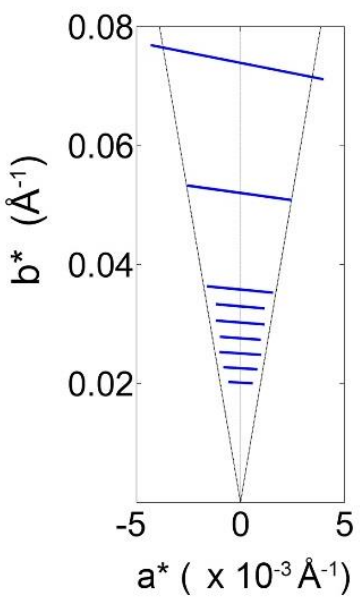

C

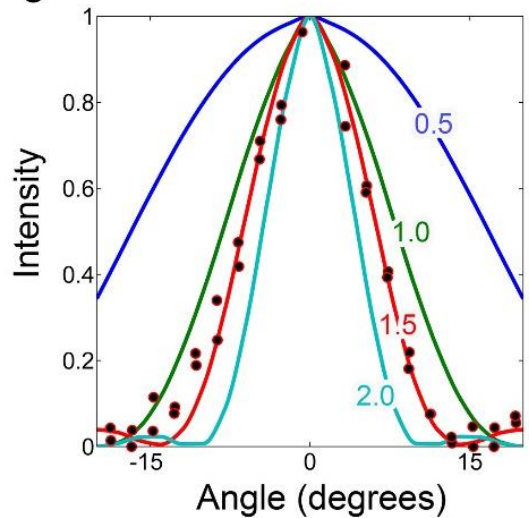

g

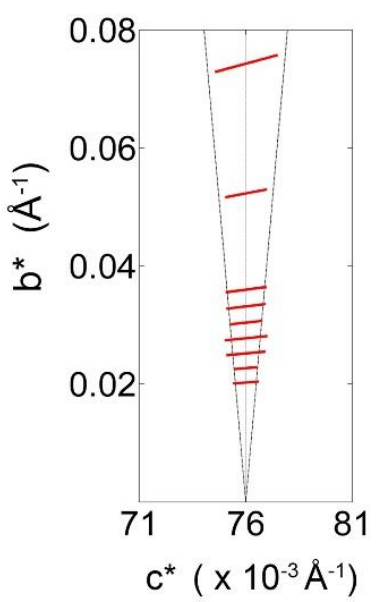

Figure 1. (a) Bragg-filtered high-resolution TEM image of MFI nanosheet along the [010] direction with the overlaid crystal structure. (b) SAED pattern taken along [010] zone axis. It is presented in reverse gray-scale color for better visibility. Tilt-axis is indicated with a double sided orange arrow. Diffraction spots used for thickness analysis are circled. (c) Variation in intensity with tilt angle (rel-rod map) of the (301) spot. Solid lines represent multislice simulated data and black dots represent experimental data. A good agreement with 1.5 uc thick nanosheet is observed. (d) Two sine waves in aand c-direction, the superposition of which provides the best description of the wrinkles in the MFI nanosheet. (e) Bragg filtered HR-TEM image shown in (a) overlaid with the estimated wrinkled nanosheet model. (f) $\mathrm{a}^{*} \mathrm{~b}^{*}$ and $(\mathrm{g}) \mathrm{b}^{*} \mathrm{c}^{*}$ projections of the (011) rel-rod constructed from experimental SAED patterns of a MFI nanosheet. Ewald sphere intersections with the rel-rod are plotted as solid blue and red lines. The cones determine the broadening of the rel-rods due to nanosheet wrinkling. 